\title{
On Some Bilateral Generating Relations Involving I-Function
}

\author{
Gauri Shankar Pandey \\ Govt. Science College, Rewa (m. P.)
}

Abstract: The aim of this research paper is to establish some bilateral generating relations involving I-function of two variables.

\section{Introduction}

The I-function of two variables introduced by Sharma \& Mishra [2], will be defined and represented as follows:

$$
\begin{aligned}
& I\left[\begin{array}{l}
x \\
y
\end{array}\right]=\left.I_{p_{i}, q_{i}: r: p_{i^{\prime}}{ }^{\prime}, q_{i}{ }^{\prime}, r^{\prime}: p_{i}^{\prime \prime}, q_{i}{ }^{\prime \prime}: r^{\prime \prime}}^{0, n: m_{y}}\right|_{\left[\left(b_{j i}: \beta_{j i}, B_{j i}\right)_{1, q_{i}}\right]} ^{\left[\left(a_{j}: \alpha_{j}, A_{j}\right)_{1, n}\right],\left[\left(a_{j i}: \alpha_{j i}, A_{j i}\right)_{n}+1, p_{i}\right]}
\end{aligned}
$$

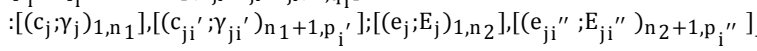

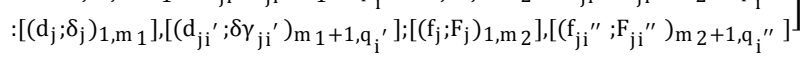

$$
\begin{aligned}
& =\frac{1}{(2 \pi \omega)^{2}} \int_{L_{1}} \int_{L_{2}} \phi_{1}(\xi, \eta) \theta_{2}(\xi) \theta_{3}(\eta) x^{\xi} y^{\eta} d \xi d \eta \text {, }
\end{aligned}
$$

where

$$
\begin{aligned}
& \phi_{1}(\xi, \eta)=\frac{\prod_{j=1}^{n} \Gamma\left(1-a_{j}+\alpha_{j} \xi+A_{j} \eta\right)}{\sum_{i=1}^{r}\left[\prod_{j=n+1}^{p_{i}} \Gamma\left(a_{j i}-\alpha_{j i} \xi-A_{j i} \eta\right) \prod_{j=1}^{q_{i}} \Gamma\left(1-b_{j i}+\beta_{j i} \xi+B_{j i} \eta\right)^{\prime}\right.},
\end{aligned}
$$

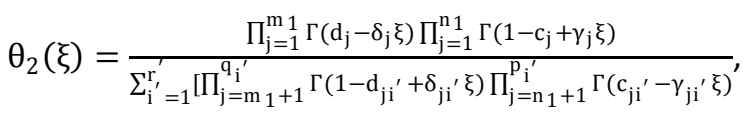

$$
\begin{aligned}
& \theta_{3}(\eta)=\frac{\prod_{j=1}^{m_{2}} \Gamma\left(f_{j}-F_{j} \eta\right) \prod_{j=1}^{n} \Gamma\left(1-e_{j}+E_{j} \eta\right)}{\sum_{i}^{r^{\prime \prime}=1}\left[\prod_{j=m_{2}+1}^{q_{i}{ }^{\prime \prime}} \Gamma\left(1-f_{j i}{ }^{\prime \prime}+F_{j i}{ }^{\prime \prime} \eta\right) \prod_{j=n_{2}+1}^{p_{i}{ }^{\prime \prime}} \Gamma\left(e_{j i}{ }^{\prime \prime}-E_{j i}{ }^{\prime \prime} \eta\right)\right.},
\end{aligned}
$$

$x$ and $y$ are not equal to zero, and an empty product is interpreted as unity $p_{i}, p_{i^{\prime}}, p_{i^{\prime \prime}}, q_{i}, q_{i^{\prime}}, q_{i^{\prime \prime}}, n, n_{1}$, $n_{2}, n_{j}$ and $m_{k}$ are non negative integers such that $p_{i} \geq n \geq 0, p_{i^{\prime}} \geq n_{1} \geq 0, p_{i^{\prime \prime}} \geq n_{2} \geq 0, q_{i}>0, q_{i^{\prime}} \geq 0, q_{i^{\prime \prime}} \geq 0$, $\left(i=1, \ldots, r ; i^{\prime}=1, \ldots, r^{\prime} ; i^{\prime \prime}=1, \ldots, r^{\prime \prime} ; k=1,2\right)$ also all the A's, $\alpha$ 's, B's, $\beta$ 's, $\gamma$ 's, $\delta$ 's, E's and F's are assumed to be positive quantities for standardization purpose; the definition of I-function of two variables given above will however, have a meaning even if some of these quantities are zero. The contour $L_{1}$ is in the $\xi$-plane and runs from $-\omega \infty$ to $+\omega \infty$, with loops, if necessary, to ensure that the poles of $\Gamma\left(d_{j}-\delta_{j} \xi\right)\left(j=1, \ldots \ldots \ldots, m_{1}\right)$ lie to the right, and the poles of $\Gamma\left(1-c_{j}+\gamma_{j} \xi\right)\left(j=1, \ldots, n_{1}\right), \Gamma\left(1-a_{j}+\right.$ $\left.\alpha_{j} \xi+A_{j} \eta\right)(j=1, \ldots, n)$ to the left of the contour.

The contour $L_{2}$ is in the $\eta$-plane and runs from $-\omega \infty$ to $+\omega \infty$, with loops, if necessary, to ensure that the poles of $\Gamma\left(f_{j}-F_{j} \eta\right) \quad\left(j=1, \ldots . ., n_{2}\right)$ lie to the right, and the poles of $\Gamma\left(1-e_{j}+E_{j} \eta\right)(j=1, \ldots$, $\left.m_{2}\right), \Gamma\left(1-a_{j}+\alpha_{j} \xi+A_{j} \eta\right)(j=1, \ldots, n)$ to the left of the contour. Also

$$
\begin{gathered}
R^{\prime}=\sum_{\mathrm{j}=1}^{\mathrm{p}_{\mathrm{i}}} \alpha_{\mathrm{ji}}+\sum_{\mathrm{j}=1}^{\mathrm{p}_{\mathrm{i}}{ }^{\prime}} \gamma_{\mathrm{ji}}{ }^{\prime}-\sum_{\mathrm{j}=1}^{\mathrm{q}_{\mathrm{i}}} \beta_{\mathrm{ji}}-\sum_{\mathrm{j}=1}^{\mathrm{q}_{\mathrm{i}}{ }^{\prime}} \delta_{\mathrm{ji}}{ }^{\prime}<0, \\
S^{\prime}=\sum_{\mathrm{j}=1}^{\mathrm{p}_{\mathrm{i}}} \mathrm{A}_{\mathrm{ji}}+\sum_{\mathrm{j}=1}^{\mathrm{p}_{\mathrm{i}}{ }^{\prime \prime}} \mathrm{E}_{\mathrm{ji}}{ }^{\prime \prime}-\sum_{\mathrm{j}=1}^{\mathrm{q}_{\mathrm{i}}} \mathrm{B}_{\mathrm{ji}}-\sum_{\mathrm{j}=1}^{\mathrm{q}_{\mathrm{i}}{ }^{\prime \prime}} \mathrm{F} \delta_{\mathrm{ji}}{ }^{\prime}<0, \\
U^{\prime}=\sum_{\mathrm{j}=\mathrm{n}+1}^{\mathrm{p}_{\mathrm{i}}} \alpha_{\mathrm{ji}}-\sum_{\mathrm{j}=1}^{\mathrm{q}_{\mathrm{i}}} \beta_{\mathrm{ji}}+\sum_{\mathrm{j}=1}^{\mathrm{m}_{1}} \delta_{\mathrm{j}}-\sum_{\mathrm{j}=\mathrm{m}_{1}+1}^{\mathrm{q}_{\mathrm{i}}{ }^{\prime}} \delta_{\mathrm{ji}}{ }^{\prime}+\sum_{\mathrm{j}=1}^{\mathrm{n}_{1}} \gamma_{\mathrm{j}}-\sum_{\mathrm{j}=\mathrm{n}_{1}+1}^{\mathrm{p}^{\prime}{ }^{\prime}} \gamma_{\mathrm{ji}}{ }^{\prime}>0, \\
V^{\prime}=-\sum_{\mathrm{j}=\mathrm{n}+1}^{\mathrm{p}_{\mathrm{i}}} \mathrm{A}_{\mathrm{ji}}-\sum_{\mathrm{j}=1}^{\mathrm{q}_{\mathrm{i}}} \mathrm{B}_{\mathrm{ji}}-\sum_{\mathrm{j}=1}^{\mathrm{m}_{2}} \mathrm{~F}_{\mathrm{j}}-\sum_{\mathrm{j}=\mathrm{m}_{21}+1}^{\mathrm{q}_{\mathrm{i}}{ }^{\prime \prime}} \mathrm{F}_{\mathrm{ji}}{ }^{\prime \prime}+\sum_{\mathrm{j}=1}^{\mathrm{n}_{2}} \mathrm{E}_{\mathrm{j}}-\sum_{\mathrm{j}=\mathrm{n}_{2}+1}^{\mathrm{p}^{\prime \prime}} \mathrm{E}_{\mathrm{ji}}{ }^{\prime \prime}>0, \\
\text { and }|\arg \mathrm{x}|<1 / 2 U^{\prime} \pi,|\arg \mathrm{y}|<1 / 2 V^{\prime} \pi .
\end{gathered}
$$

In the present investigation we require the following formulae:

From Rainvile [1, p.93]:

$$
{ }_{2} F_{1}\left[\begin{array}{c}
-n, a ; \\
1+a+n ;
\end{array}-1\right]=\frac{(1+a)_{n}}{(1+a / 2)_{n}},
$$


From Shrivastava and Manocha [3, p.37 (10), 34, 44],

$$
\begin{aligned}
& (\alpha)_{n}=(\alpha, n)=\frac{\Gamma(\alpha+n)}{\Gamma(\alpha)}, \\
& (1-\mathrm{z})^{-\mathrm{a}}=\sum_{n=0}^{\infty}(a)_{n} \frac{z^{n}}{n !},
\end{aligned}
$$

\section{Bilateral Generating Relations}

In this section we establish the following bilateral Generating Relations:

$$
\begin{aligned}
& \sum_{n=0}^{\infty} \frac{\mathrm{t}^{n}}{n !}{ }_{2} \mathrm{~F}_{1}\left[\begin{array}{c}
-\mathrm{n}, \mathrm{a} ; \\
1+\mathrm{a}+\mathrm{n} ;
\end{array}-1\right]
\end{aligned}
$$

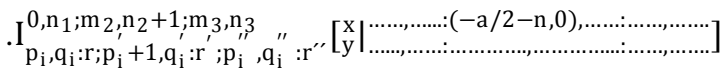

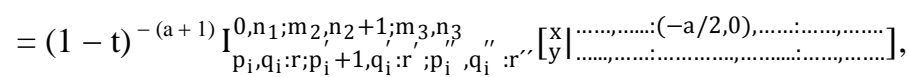

provided that $\mathrm{U}^{\prime}>0, V^{\prime}>0,|\operatorname{argx}|<\frac{1}{2} \mathrm{U}^{\prime} \pi,|\operatorname{argy}|<\frac{1}{2} \mathrm{~V}^{\prime} \pi$ where $\mathrm{U}^{\prime}$ and $\mathrm{V}^{\prime}$ are given in (2) and (3).

$$
\begin{aligned}
& \sum_{n=0}^{\infty} \frac{\mathrm{t}^{n}}{n !} \quad{ }_{2} \mathrm{~F}_{1}\left[\begin{array}{l}
-\mathrm{n}, \mathrm{a} ; \\
1+\mathrm{a}+\mathrm{n} ;
\end{array}-1\right]
\end{aligned}
$$

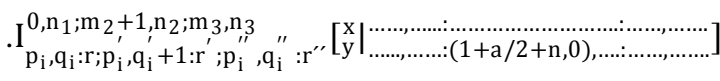

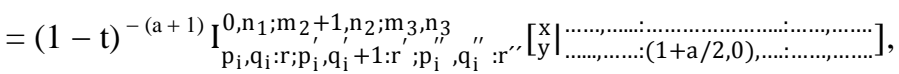

provided that $\mathrm{U}^{\prime}>0, V^{\prime}>0,|\operatorname{argx}|<\frac{1}{2} \mathrm{U}^{\prime} \pi,|\operatorname{argy}|<\frac{1}{2} \mathrm{~V}^{\prime} \pi$ where $\mathrm{U}^{\prime}$ and $\mathrm{V}^{\prime}$ are given in (2) and (3).

Proof:

To prove (7), consider

$$
\begin{aligned}
& \Delta=\sum_{n=0}^{\infty} \frac{\mathrm{t}^{n}}{n !} \quad{ }_{2} \mathrm{~F}_{1}\left[\begin{array}{c}
-\mathrm{n}, \mathrm{a} ; \\
1+\mathrm{a}+\mathrm{n} ;
\end{array}-1\right]
\end{aligned}
$$

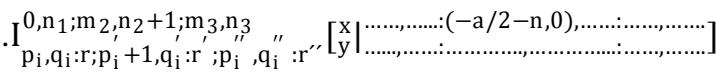

On expressing I-function in contour integral form as given in (1) and using (4), we get

$$
\begin{aligned}
\Delta & =\sum_{n=0}^{\infty} \frac{\mathrm{t}^{n}}{n !} \frac{(1+\mathrm{a})_{\mathrm{n}}}{(1+\mathrm{a} / 2)_{\mathrm{n}}} \\
& \frac{1}{(2 \pi \omega)^{2}} \int_{L_{1}} \int_{L_{2}} \phi_{1}(\xi, \eta) \theta_{2}(\xi) \theta_{3}(\eta) \Gamma\left\{1-\left(-\frac{\mathrm{a}}{2}-\mathrm{n}\right)+0 \xi\right\} x^{\xi} y^{\eta} d \xi d \eta
\end{aligned}
$$

In the view of (5) and (6), we arrive at R.H.S. of (7) as follows:

$$
\begin{gathered}
\Delta=\sum_{n=0}^{\infty} \frac{\mathrm{t}^{n}}{n !} \frac{(1+\mathrm{a})_{\mathrm{n}}}{(1+\mathrm{a} / 2)_{\mathrm{n}}} \\
\cdot \frac{1}{(2 \pi \omega)^{2}} \int_{L_{1}} \int_{L_{2}} \phi_{1}(\xi, \eta) \theta_{2}(\xi) \theta_{3}(\eta)\left(1+\frac{\mathrm{a}}{2}\right)_{\mathrm{n}} \Gamma(1+\mathrm{a} / 2) x^{\xi} y^{\eta} d \xi d \eta \\
=\frac{1}{(2 \pi \omega)^{2}} \int_{L_{1}} \int_{L_{2}} \phi_{1}(\xi, \eta) \theta_{2}(\xi) \theta_{3}(\eta) \Gamma(1+\mathrm{a} / 2) \\
\cdot\left[\sum_{n=0}^{\infty} \frac{\mathrm{t}^{n}}{n !}(1+\mathrm{a})_{\mathrm{n}}\right] x^{\xi} y^{\eta} d \xi d \eta
\end{gathered}
$$




$$
\begin{aligned}
& =\frac{1}{(2 \pi \omega)^{2}} \int_{L_{1}} \int_{L_{2}} \phi_{1}(\xi, \eta) \theta_{2}(\xi) \theta_{3}(\eta) \\
& \Gamma(1+\mathrm{a} / 2)(1-\mathrm{t})^{-(\mathrm{a}+1)} x^{\xi} y^{\eta} d \xi d \eta
\end{aligned}
$$

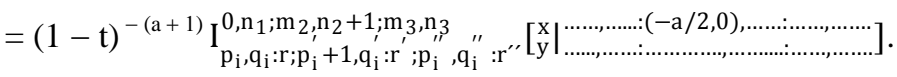

Proceeding on similar lines as above, the results (8) can be derived easily.

\section{Particular Cases}

On choosing $r=1, r^{\prime}=1$ and $r^{\prime \prime}=1$ in main integrals, we get following integrals in terms of $\mathrm{H}$-function of two variables:

$$
\begin{aligned}
& \sum_{n=0}^{\infty} \frac{\mathrm{t}^{n}}{n !}{ }_{2} \mathrm{~F}_{1}\left[\begin{array}{c}
-\mathrm{n}, \mathrm{a} ; \\
1+\mathrm{a}+\mathrm{n} ;-1]
\end{array}\right.
\end{aligned}
$$

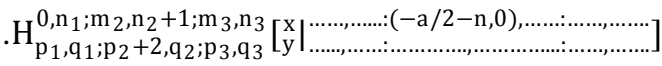

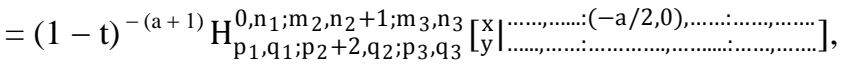

provided that $\mathrm{U}>0, V>0,|\arg \mathrm{x}|<\frac{1}{2} \mathrm{U} \pi,|\operatorname{argy}|<\frac{1}{2} \mathrm{~V} \pi$ where $\mathrm{U}$ and $\mathrm{V}$ are given by:

$$
\begin{aligned}
& U=-\Sigma^{p_{1}} \alpha_{j}-\Sigma \beta_{j}^{q_{1}}+\Sigma \delta_{j}^{m_{2}}-\Sigma \delta_{j}^{q_{2}}+\Sigma \gamma_{j}-\Sigma{\stackrel{\gamma_{j}}{\gamma_{j}}}_{>0,}^{p_{2}} \\
& \mathrm{j}=\mathrm{n}_{1}+1 \quad \mathrm{j}=1 \quad \mathrm{j}=1 \quad \mathrm{j}=\mathrm{m}_{2}+1 \quad \mathrm{j}=1 \quad \mathrm{j}=\mathrm{n}_{2}+1 \\
& V=-\Sigma^{p_{1}} A_{j}-\Sigma B_{j}^{q_{1}}+\Sigma F_{j}-\Sigma F_{j}+{ }^{q_{3}} \Sigma E_{j}-\Sigma^{n_{3}} E_{j} \quad>0 \text {, } \\
& j=n_{1}+1 \quad j=1 \quad j=1 \quad j=m_{3}+1 j=1 \quad j=n_{3}+1 \\
& \sum_{n=0}^{\infty} \frac{\mathrm{t}^{n}}{n !} \quad{ }_{2} \mathrm{~F}_{1}\left[\begin{array}{c}
-\mathrm{n}, \mathrm{a} ; \\
1+\mathrm{a}+\mathrm{n} ;
\end{array}-1\right]
\end{aligned}
$$

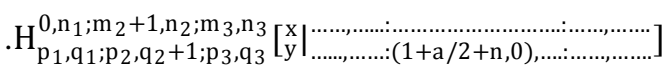

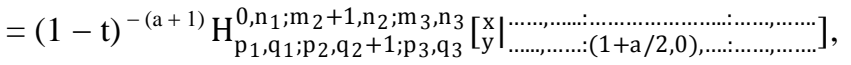

provided that $U>0, V>0,|\operatorname{argx}|<\frac{1}{2} U \pi,|\operatorname{argy}|<\frac{1}{2} \mathrm{~V} \pi$ where $U$ and $V$ are given in (10) and (11).

\section{References}

[1]. Rainville, E. D.: Special Functions, Macmillan, NewYork, 1960.

[2]. Sharma C. K. and Mishra, P. L.: On the I-function of two variables and its certain properties, ACI, 17 (1991), 1-4.

[3]. Shrivastava, H. M. and Manocha, H. L.: A treatise on generating functions, Ellis Horwood Limited England. 